\title{
Excitation of the Synchro-Betatron Resonances by the Beam-Beam Interaction in the Tevatron Run II Lattice
}

\author{
Y.Alexahin* \\ Fermi National Accelerator Laboratory \\ P.O. Box 500, Batavia, Illinois 60510
}

\begin{abstract}
Effect of different mechanisms of the synchro-betatron coupling (dispersion function at the interaction points, chromatic tune modulation, finite bunch length) on the p-bar stability in the Tevatron Run II configuration is considered. It is found that the long-range interactions in the presence of large dispersion produce large contribution $(\sim 10)$ to the chromaticity of the betatron tunes. This chromaticity, in its turn, can give rise to multiple synchrotron satellites of the betatron resonances increasing their effective width.

Novel formulae are presented which permit to significantly speed up analytical calculations of the beam-beam tuneshifts and resonance strength.
\end{abstract}

April 2001

* Permanent address: JINR, Dubna 141980 Russia 


\section{Introduction}

There are several reasons owing to which the beam-beam driven resonances (the synchro-betatron ones in particular) can present in the Tevatron Run II configuration [1] a greater danger than in the previous runs. Besides some increase in the beam-beam tuneshift due to a larger number of protons per bunch and a smaller proton emittance (see Table 1), there will be a factor of seven larger number of the parasitic long-range interactions, some of them at only $\sim 7 \sigma_{\perp}$ separation (see Fig.1) with the Run II lattice version available on the Web [2]. These numerous parasitic encounters will strongly enhance excitation of the odd-order resonances.

\begin{tabular}{|ll|r|r|r|}
\hline & Run Ib & Run II & Run II, SC RF \\
\hline$N_{p}$ /bunch, & $10^{11}$ & 2.32 & 2.7 & 2.7 \\
\hline$\varepsilon_{p}$ (95\% normal.), & $\pi \cdot \mathrm{mm} \cdot \mathrm{mrad}$ & 23 & 20 & 20 \\
\hline$\xi$ /nominal IP & 0.0074 & 0.01 & 0.01 \\
\hline$N$ parasitic IPs & 10 & 70 & 70 \\
\hline$\xi$, total & $\sim 0.015$ & $\sim 0.025$ & $\sim 0.025$ \\
\hline$\sigma_{s}$, & 63 & 37 & 14 \\
\hline$\sigma_{E}$, & 1.5 & 0.9 & 3.1 \\
\hline $\mathrm{v}_{s}$, & $\mathrm{cm}$ & 0.7 & 0.7 & 6.4 \\
\hline
\end{tabular}

Table 1. Comparison of the proton beam parameters for different Tevatron runs.

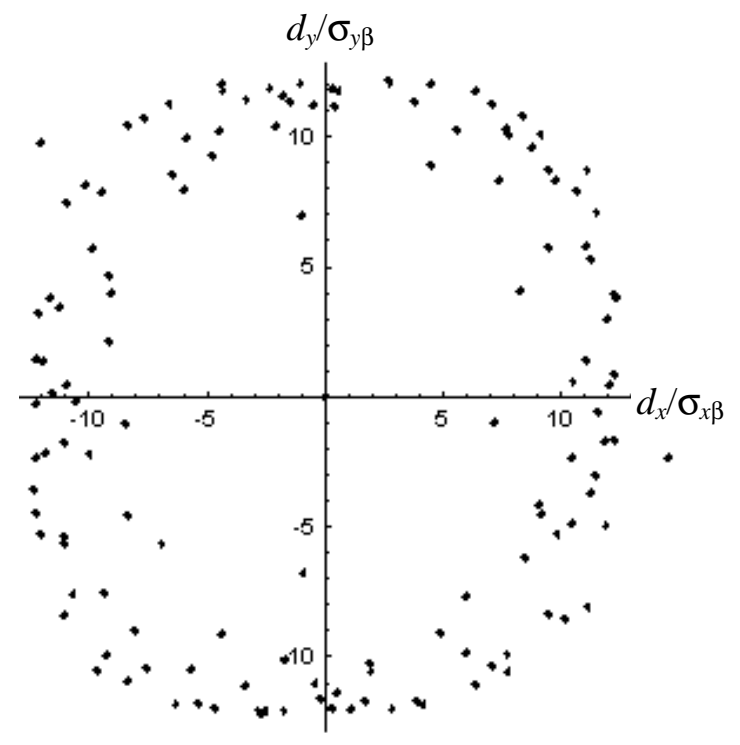

Figure 1. Normalized beam separation at all possible interaction points with $36 \times 36$ bunches.
Another important effect which the long-range interactions give rise to is the beam-beam induced chromaticity (observed experimentally at LEP [3]). It depends on the particle transverse amplitudes and the bunch collision schedule and therefore can not be eliminated with the help of sextupoles. The large chromatic tune modulation of the off-momentum particles will produce synchrotron satellites increasing the effective width of the betatron resonances. There is also an additional contribution to the satellite excitation owing to the finite dispersion at the location of parasitic encounters.

The effect of the head-on interactions can be more pronounced in the Run II configuration as well due to a smaller bunch length (see Table 1), especially with introduction of the superconducting $\mathrm{RF}$ which will provide $V_{\mathrm{RF}}=20 \mathrm{MV}$ at $212 \mathrm{MHz}$ as compared to $V_{\mathrm{RF}}=1 \mathrm{MV}$ at $53 \mathrm{MHz}$ with the existing RF system. The reduction in the bunch length is big enough to weaken

the beneficial phase averaging effect $[4,5,6]$ but may be insufficient to avoid excitation of the synchrotron satellites by the betatron phase modulation of the beam-beam kicks at the low-beta interaction points (IPs).

The present report is devoted to the analytical study of the beam-beam driven synchro-betatron resonances in the Run II configuration and to some development of the theoretical tools which occurred necessary to perform this study. 


\section{Beam-beam potential}

Let us choose the generalized azimuth $\theta=s / R, R$ being the mean radius of the orbit, as the independent variable (so that the longitudinal momentum plays the role of the Hamiltonian) and introduce canonically conjugated coordinates and momenta $\underline{q}=(x, y, \sigma), \underline{p}=\left(p_{x}, p_{y}, \delta_{p}\right)$, where in the ultrarelativistic case $\sigma=s-c t$ for one beam (let it be p-bar) and $\sigma=-s-c t$ for the other.

Due to the synchrotron motion the actual collision point (CP) of two particles belonging to the counter-rotating bunches is shifted w.r.t. the interaction point (IP) of the reference particles by the amount

$$
s_{\mathrm{CP}}-s_{\mathrm{IP}}=\frac{\sigma_{1}-\sigma_{2}}{2} .
$$

If the r.m.s. bunch length $\sigma_{s}$ is comparable with the beta-function this shift leads to: i) modulation of the betatron phase by synchrotron motion thus provoking SBRs; ii) phase averaging of high order betatron resonances.

To analyze these effects (well-known in principle) we will apply here a technique permitting to lump the interaction with a long bunch to a single point.

It was shown in Ref.[7] how to ascribe the interaction which takes place at a shifted point to the position of the reference IP with the help a similarity transformation and construct a symplectic "synchro-beam" map. The generating Hamiltonian for this map can be found [8] and presented in the form

$$
H_{b b}=-\frac{r_{p} N}{\gamma} \delta_{\mathrm{p}}\left(\theta-\theta_{I P}\right) \int G\left(\underline{q}, \underline{p} \mid \underline{q^{\prime}}, \underline{p^{\prime}}\right) F_{0}\left(\underline{q^{\prime}}, \underline{p^{\prime}}, \theta\right) d^{3} q^{\prime} d^{3} p^{\prime},
$$

where $\delta_{\mathrm{p}}(\theta)$ is the periodic $\delta$-function, $N$ is the number of particles in the bunch, $F_{0}$ is the normalized to unity distribution function, $r_{p}$ is the classical radius of the particles, $\gamma$ is the relativistic mass factor, $G$ is Green's function.

In the case of a finite horizontal crossing angle, $2 \alpha$, Green's function can be written in the form

$$
G=-\ln \left\{\left[x-x^{\prime}+\left(\alpha+\frac{p_{x}+p_{x}^{\prime}}{2}\right)\left(\sigma-\sigma^{\prime}\right)\right]^{2}+\left[y-y^{\prime}+\frac{p_{y}+p_{y}^{\prime}}{2}\left(\sigma-\sigma^{\prime}\right)\right]^{2}\right\},
$$

its dependence on the momenta being the price paid for lumping all the interaction at the point $\theta=\theta_{I P}$.

Let us assume that the particle motion in the strong (proton) beam is regular so that invariants of motion $\underline{I}=\left(I_{x}, I_{y}, I_{s}\right)$ exist. They can be used in the construction of the equilibrium distribution function which we presume to be Gaussian:

$$
F_{0}=\frac{1}{(2 \pi)^{3} V} \exp \left(-\underline{\varepsilon^{-1}} \cdot \underline{I}\right), \quad \underline{\varepsilon}=\langle\underline{I}\rangle, \quad V=\varepsilon_{x} \varepsilon_{y} \varepsilon_{s}, \quad \underline{\varepsilon^{-1}}=\left(\varepsilon_{x}^{-1}, \varepsilon_{y}^{-1}, \varepsilon_{s}^{-1}\right) .
$$

where $\varepsilon_{i}, i=x, y, s$, are the r.m.s. emittances (mean values of the action variables).

To evaluate the beam-beam potential (2.2) let us express the coordinates and momenta via the action-angle variables. First we extract the closed orbit and dispersion 


$$
\begin{aligned}
& x=x_{\mathrm{co}}+D_{x} \delta_{p}+\tilde{x}, \quad p_{x}=x_{\mathrm{co}}^{\prime}+\left(D_{x}^{\prime}+x_{\mathrm{co}}^{\prime}\right) \delta_{p}+\tilde{p}_{x}, \\
& \sigma=\tilde{p}_{x} D_{x}-\tilde{x}\left(D_{x}^{\prime}+x_{\mathrm{co}}^{\prime}\right)+\tilde{p}_{y} D_{y}-\tilde{y}\left(D_{y}^{\prime}+y_{\mathrm{co}}^{\prime}\right)+\tilde{\sigma},
\end{aligned}
$$

where the tilde marks variables describing betatron and synchrotron oscillations (uncoupled in the linear approximation) and prime denotes differentiation by $s$. The most important nonlinear term for the subsequent analysis arises from the betatron tune modulation by the synchrotron motion (see Ref.[8] for the rigorous introduction via a Lie-transform)

$$
\begin{aligned}
& \tilde{x}=\sqrt{2 \beta_{x} I_{x}} \sin \left(\psi_{x}+\phi_{x}-\eta_{x} \tilde{\sigma}\right), \\
& \tilde{p}_{x}=\sqrt{2 I_{x} / \beta_{x}}\left[\cos \left(\psi_{x}+\phi_{x}-\eta_{x} \tilde{\sigma}\right)-\alpha_{x} \sin \left(\psi_{x}+\phi_{x}-\eta_{x} \tilde{\sigma}\right)\right], \\
& \tilde{\sigma}=\sqrt{2 \beta_{s} I_{s}} \sin \psi_{s}, \quad \delta_{p}=\sqrt{2 I_{s} / \beta_{s}} \cos \psi_{s},
\end{aligned}
$$

where $\alpha_{x}=-\beta_{x}{ }^{\prime} / 2$ is the Twiss parameter, $\beta_{s}=R \alpha_{M} / v_{s}$ is the longitudinal $\beta$-function with $\alpha_{M}$ being the momentum compaction factor,

$$
\phi_{x}(\theta)=\int_{0}^{R \theta} \frac{d s^{\prime}}{\beta_{x}}-v_{x 0} \theta
$$

is the periodic betatron phase function and

$$
\eta_{x}=\frac{1}{\alpha_{M} R} \frac{d \nu_{x}}{d \delta_{p}} .
$$

The formulas for the vertical plane are analogous to (2.5-8).

Let us note that parameters $\alpha_{x}, x_{\mathrm{co}}{ }^{\prime}, D_{x}^{\prime}$ entering Green's function (2.3) via momenta can be important only for low-beta interaction points where they should be zero (in an absence of misalignments). Therefore we may put $\alpha_{x}=x_{\mathrm{co}}{ }^{\prime}=D_{x}{ }^{\prime}=0$ everywhere (and so for the vertical plane). Also, we make a number of assumptions to simplify the formulae:

1) the betatron functions at the low-beta IPs to be equal, $\beta_{\mathrm{x}}{ }^{*}=\beta_{\mathrm{y}}{ }^{*}=\beta^{*}$, so that the finite bunch length effect in both planes is described by the same variable

$$
b=\frac{\sigma-\sigma^{\prime}}{2 \beta^{*}},
$$

2) the dispersion functions for both beams are equal (which is not really so in Tevatron);

3) the vertical dispersion function and crossing angle are negligible.

Now let us introduce the r.m.s. beam sizes of the strong bunch and their ratio $r$ :

$$
\begin{aligned}
& \sigma_{i}=\sqrt{\varepsilon_{i} \beta_{i}}, \quad i=x, y, s, \\
& \sigma_{x t}=\sigma_{x} \sqrt{1+\kappa^{2}}, \\
& \kappa=\sqrt{\sigma_{x}^{2}+\alpha^{2} \sigma_{s}^{2}+D_{x}^{2} \sigma_{E}^{2}} / \sigma_{x}, \quad \sigma_{E}=\sigma_{s} / \beta_{s}, \\
& r=\sigma_{y} / \sigma_{x t} .
\end{aligned}
$$


Representing Green's function as a Fourier transform

$$
G=-\ln \left(\Delta x^{2}+\Delta y^{2}\right)=\frac{r}{\pi} \int_{-\infty-\infty}^{\infty} \int_{\infty}^{\infty} \exp \left(i k_{1} \frac{\Delta x}{\sigma_{x t}}+i k_{2} \frac{\Delta y}{\sigma_{y}}\right) \frac{d k_{1} d k_{2}}{r^{2} k_{1}^{2}+k_{2}^{2}}
$$

and performing in (2.2) integration by the transverse variables we obtain

$$
\begin{aligned}
H_{b b}= & -\frac{r_{p} N}{\pi \gamma} \delta_{\mathrm{p}}\left(\theta-\theta_{I P}\right) \iint \frac{r d u_{1} d u_{2}}{r^{2} u_{1}^{2}+u_{2}^{2}} \exp \left[-\frac{u_{1}^{2} \sigma_{x}^{2}}{2 \sigma_{x t}^{2}}-\frac{u_{2}^{2}}{2}\right] \iint d \sigma^{\prime} d \delta_{p}^{\prime} F_{L}\left(\sigma^{\prime}, \delta_{p}^{\prime}\right) \times \\
& \exp \left\{i \frac{u_{1}}{\sigma_{x t}}\left[\frac{d_{x}+D_{x}\left(\delta_{p}-\delta_{p}^{\prime}\right)+\alpha\left(\sigma-\sigma^{\prime}\right)}{\sqrt{1+b^{2}}}+\tilde{\tilde{x}}\right]+i \frac{u_{2}}{\sigma_{y}}\left[\frac{d_{y}}{\sqrt{1+b^{2}}}+\tilde{\tilde{y}}\right]\right\}
\end{aligned}
$$

where $F_{L}$ is the longitudinal distribution function (normalized to unity), $u_{i}=k_{i}\left(1+b^{2}\right)^{1 / 2}$, double tilde denotes additional phase shift due to displacement (2.1) of the actual collision point:

$$
\tilde{\tilde{x}}=\exp \left[-i \varphi \frac{\partial}{\partial \psi_{x}}\right] \tilde{x}, \quad \varphi=-\arctan b .
$$

In the following we will neglect the variable $b$ appearing anywhere else in eq.(2.12) besides the phase $\varphi$. This is justified in the absence of large crossing angle $\alpha$ since at the nominal IPs, where $b$ can reach appreciable values, parameters $d_{x, y}, D_{x}$ should be zero (in the absence of imperfections) whereas at parasitic IPs $b$ is small. Let us note, in passing, that the terms we are going to neglect provide extra suppression of the effect of imperfections in addition to the phase averaging described by $\varphi$.

When the finite bunch length effect can be ignored altogether, the beam-beam potential can be reduced to the form

$$
\begin{aligned}
H_{b b}= & -\frac{2 r_{p} N}{\gamma} \delta_{\mathrm{p}}\left(\theta-\theta_{I P}\right) \int_{0}^{1} \frac{d t}{t \sqrt{1+\left(r^{2}-1\right) t^{2}}} \times \\
& \exp \left\{-\frac{\left(d_{x}+\tilde{x}+D_{x} \delta_{p}+\alpha \widetilde{\sigma}\right)^{2}}{2 \sigma_{x t}^{2}} t^{2}-\frac{\left(d_{y}+\tilde{y}\right)^{2}}{2 \sigma_{y}^{2}\left[1+\left(r^{2}-1\right) t^{2}\right]} r^{2} t^{2}\right\}
\end{aligned}
$$

which was the starting point for analysis in Ref.[9] ${ }^{1}$. In its derivation we made use of the formula

$$
\frac{1}{r^{2} u_{1}^{2}+u_{2}^{2}}=\int_{0}^{\infty} e^{-\left(r^{2} u_{1}^{2}+u_{2}^{2}\right) \tau} d \tau
$$

with subsequent integration by the longitudinal variables and $u_{1,2}$ and finally setting $\tau=\left(t^{-2}-1\right) / 2 r^{2}$.

\footnotetext{
${ }^{1}$ In the Mathematica notebook inc_bb.nb cited in Ref.[9] the sign of the potential had been chosen opposite to that in eq.(2.14) to describe the $p-p$ interaction. Also, there was a minor misprint in the formula not affecting the actual calculations. Let us note that a similar representation of the beam-beam potential was used by J.Jowett [10].
} 


\section{Beam-beam contribution to the tunes and chromaticity}

The beam-beam tuneshifts are defined by the formula

$$
\Delta \boldsymbol{v}_{i}^{(b b)}=\frac{\partial}{\partial I_{i}}\left\langle H_{b b}\right\rangle, i=x, y, s, .
$$

where the brackets denote averaging over all phase variables, $\psi_{i}$, and the azimuth, $\theta$. The averaging eliminates dependence on the phase $\varphi$, so there is no finite bunch length effect on the tunes under the assumptions made (equal $\beta$-functions and small offsets and dispersion at low-beta IPs, large $\beta$ functions at parasitic IPs).

In the simplest case $\alpha=d_{x, y}=D_{x}=0$ averaging over $\psi_{i}$ can be done analytically.

Normalizing the action variables of the weak beam particles by the r.m.s. emittances of the strong beam:

$$
J_{i}=I_{i} / \varepsilon_{i}, \quad i=x, y, s,
$$

and introducing the beam-beam parameter

$$
\xi_{x}=\frac{r_{p} N}{2 \pi \gamma \varepsilon_{x}\left(1+\kappa^{2}\right)(1+r)}
$$

$(\kappa=0$ for now $)$ we obtain the formula

$$
\begin{aligned}
\Delta v_{x}^{(b b)}\left(J_{x}, J_{y}\right)=\xi_{x} & \int_{0}^{1 / 2} \frac{(1+r) d u}{\sqrt{1+2\left(r^{2}-1\right) u}} \exp \left[-J_{x} u-J_{y} \frac{r^{2} u}{1+2\left(r^{2}-1\right) u}\right] \times \\
& {\left[\mathrm{I}_{0}\left(J_{x} u\right)-\mathrm{I}_{1}\left(J_{x} u\right)\right] \cdot \mathrm{I}_{0}\left[J_{y} \frac{r^{2} u}{1+2\left(r^{2}-1\right) u}\right], }
\end{aligned}
$$

which was given without derivation in Ref.[11] and, in the case of a round beam, $r=1$, in Ref.[12].

In the general case the averaging over $\psi_{i}$ can be performed quite efficiently by direct numerical integration as proposed in Ref.[9]. We will employ this method in the present study as well. Let us remind the expression for the averaged beam-beam Hamiltonian

$$
\begin{aligned}
&\left\langle H_{b b}\right\rangle=-\frac{r_{p} N}{\gamma \pi^{4}} \int_{0}^{1} X\left[0,0,0, t \delta x, t \sqrt{\frac{J_{x}}{1+\kappa^{2}}}, t \sqrt{\frac{\kappa^{2} J_{s}}{1+\kappa^{2}}}\right] \times \\
& Y\left[0,0, \frac{r t \delta y}{\sqrt{1+\left(r^{2}-1\right) t^{2}}}, \frac{r t \sqrt{J_{y}}}{\sqrt{1+\left(r^{2}-1\right) t^{2}}}\right] \frac{d t}{t \sqrt{1+\left(r^{2}-1\right) t^{2}}},
\end{aligned}
$$

where $\delta_{x}=d_{x} / \sigma_{x t} \sqrt{ } 2, \delta_{y}=d_{y} / \sigma_{y} \sqrt{ } 2$ and

$$
\begin{aligned}
& X(l, m, n, d, x, z)= \\
& \quad \int_{0}^{\pi} \int_{0}^{\pi} \sin ^{2 m} \varphi \sin ^{2 n} \psi e^{-(d+x \cos \varphi+z \cos \psi)^{2}} \mathrm{H}_{l+m+n}(d+x \cos \varphi+z \cos \psi) d \varphi d \psi, \\
& Y(l, m, d, y)=\int_{0}^{\pi} \sin ^{2 m} \varphi e^{-(d+y \cos \varphi)^{2}} \mathrm{H}_{l+m}(d+y \cos \varphi) d \varphi
\end{aligned}
$$


$\mathrm{H}_{n}(x)$ being the Hermite polynomials. Now we can calculate the tuneshifts with the help of the relation

$$
\frac{\partial}{\partial x} X(l, m, n, d, x, z)=\frac{x}{2 m+1} X(l+1, m+1, n, d, x, z)
$$

and an analogous formula for $Y$.

Fig.2 shows the footprint of the antiproton bunch \#6 in the tune diagram obtained with the standard optics [2] at the working point (WP) $v_{x 0}=20.585, v_{y 0}=20.575$. The $5^{\text {th }}, 7^{\text {th }}$ and $12^{\text {th }}$ order sum resonance lines ${ }^{2}$ are also shown. The arc lines in the footprints correspond to equidistant step 2 values of the normalized transverse amplitude

$$
a_{\perp}=\left(a_{x}^{2}+a_{y}^{2}\right)^{1 / 2}, \quad a_{x, y}=\left(\frac{2}{\varepsilon_{\bar{p}}} I_{x, y}\right)^{1 / 2}=\left(\frac{2 \varepsilon_{p}}{\varepsilon_{\bar{p}}} J_{x, y}\right)^{1 / 2} ;
$$

the radial lines being equidistant in $\arctan \left(a_{x} / a_{y}\right)$ value.

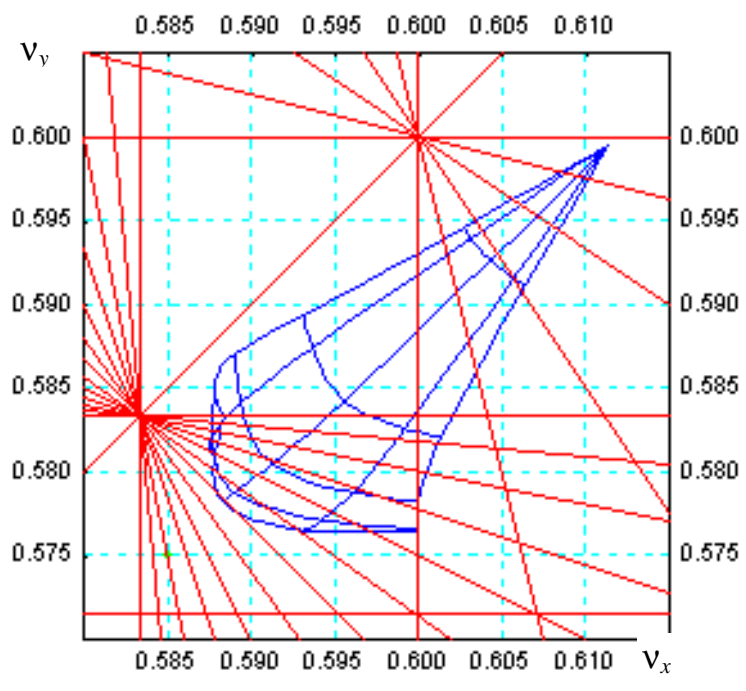

Figure 2. Footprint of the p-bar bunch \#6 in the tune diagram obtained with the optics [2]

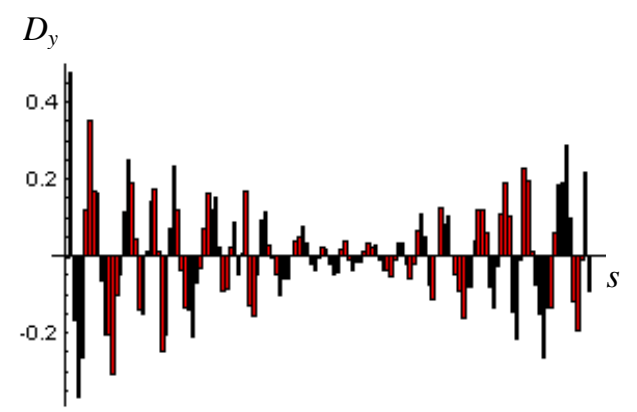

Figure 3. Vertical dispersion on the p-bar helix

Due to the finite dispersion at parasitic IPs there should be some dependence of the beam-beam tuneshifts on the synchrotron motion. Calculations for the standard Run II configuration show that for the average values of the tuneshifts over the synchrotron period this dependence is negligible.

However, there can be significant variation of the instant values of the tuneshifts which we will treat in terms of the beam-beam contribution to chromaticity. To get a feeling of the order of the effect let us consider a single long-range interaction point at horizontal separation $d_{x}$. The tuneshift for a particle with constant momentum deviation $\delta_{p}$ is

$$
\Delta \mathbf{v}_{x}^{(b b)}=-\frac{r_{p} N \beta_{x}}{4 \pi \gamma\left(d_{x}+D_{x} \delta_{p}\right)^{2}}
$$

Correspondingly, we have for the beam-beam contribution to chromaticity (which we will call just "the beam-beam chromaticity" for brevity)

$$
\frac{\partial}{\partial \delta_{p}} \Delta \mathbf{v}_{x}^{(b b)}=D_{x} \frac{\partial}{\partial d_{x}} \Delta \mathbf{v}_{x}^{(b b)}=-\frac{2 D_{x}}{d_{x}} \Delta \mathbf{v}_{x}^{(b b)}
$$

The ratio $\left|D_{x} / d_{x}\right|$ can be as large as $10^{3}$, so that for the tuneshift of the order of $10^{-3}$ the shift in chromaticity may be $\sim 1$.

Calculating the beam-beam chromaticity on the Tevatron helix one should take into account the vertical dispersion as well which can be quite large ${ }^{3}$ (see Fig.3).

\footnotetext{
2 analysis shows that the difference resonances are less important at the considered WPs.

${ }^{3} \mathrm{Still}$ its contribution to the beam sizes is not significant which justifies its exclusion from all other expressions.
} 


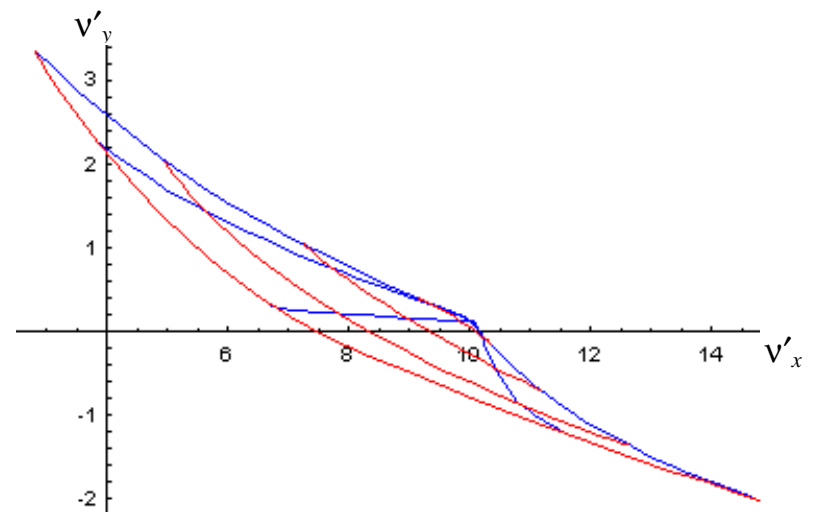

Figure 4. Footprint of the p-bar bunch \#6 in the chromaticity diagram

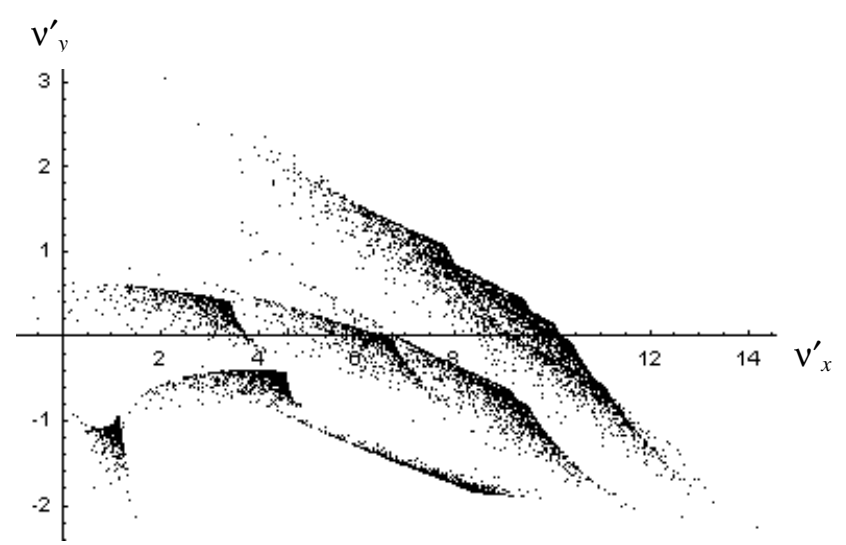

Figure 5. Total p-bar distribution in the chromaticity plane

The necessary formulas can be obtained from eq.(3.5) with account of the differentiation rule

$$
\frac{\partial}{\partial d} X(l, m, n, d, x, z)=-X(l+1, m, n, d, x, z)
$$

and its counterpart for $Y$.

Fig.4 shows the footprint of the antiproton bunch \#6 in the plane of the beam-beam chromaticities

$$
\chi_{i}\left(J_{x}, J_{y}\right)=\frac{\partial}{\partial \delta_{p}} \Delta \mathrm{v}_{i}^{(b b)}=D_{x} \frac{\partial}{\partial d_{x}} \Delta \mathrm{v}_{i}^{(b b)}+D_{y} \frac{\partial}{\partial d_{y}} \Delta \mathrm{v}_{i}^{(b b)}, \quad i=x, y,
$$

the arc lines now corresponding to the values $a_{\perp}=1,2,3,4$. The beam-beam contribution to chromaticity is really very large and strongly depends on the betatron amplitudes.

There is also significant bunch-to-bunch variation. Fig.5 shows the total distribution of antiprotons (represented by 1000 points/bunch) in the plane of chromaticities.

We see that the chromaticities can not be made small simultaneously for all particles in all bunches with the help of correction sextupoles.

\section{Beam-beam resonances}

In the present report we include the effects of the synchrotron motion into the analysis of the beambeam driven resonances in the Tevatron Run II configuration started in Ref.[9]. Since the longitudinal emittance $\varepsilon_{s}=\sigma_{s} \sigma_{E} \approx 3.5 \cdot 10^{-5} \mathrm{~m}$ is by four orders of magnitude larger than the transverse one we may ignore variation of the longitudinal action in the resonance exchange of energy and consider it as a constant of motion:

$$
I_{s}=\text { const. }
$$

Again we limit ourselves to a single resonance approximation considering one resonance at a time and then applying the Chirikov criterion to access the cooperative effect of multiple resonances.

In the vicinity of the resonance ${ }^{4}$

$$
\Delta_{\underline{m}}=\underline{m} \cdot \underline{v}-n \equiv m_{x} v_{x}+m_{y} v_{y}+m_{s} v_{s}-n \approx 0
$$

we can transform to the rotating frame and write the main terms in the Hamiltonian in the form

\footnotetext{
${ }^{4}$ Here the synchrotron tune should be understood as an algebraic value negative above the transition energy.
} 


$$
H \approx \int^{I_{1}} \Delta_{\underline{m}}\left(I_{1}^{\prime}, I_{2}, I_{s}\right) d I_{1}^{\prime}+C_{\underline{m}}\left(I_{1}, I_{2}, I_{s}\right) \cos \left(\psi_{1}+\psi_{0}\right)
$$

where the new transverse action-angle variables were introduced

$$
\begin{aligned}
& I_{1}=\frac{m_{x} I_{x}+m_{y} I_{y}}{m_{x}^{2}+m_{y}^{2}}, \quad I_{2}=\frac{m_{y} I_{x}-m_{x} I_{y}}{m_{x}^{2}+m_{y}^{2}}=\mathrm{const}, \\
& \psi_{1}=m_{x} \psi_{x}+m_{y} \psi_{y}+m_{s} \psi_{s}-n \theta, \quad \psi_{2}=m_{y} \psi_{x}-m_{x} \psi_{y},
\end{aligned}
$$

the resonance coefficient $C_{\underline{m}}$ and phase $\psi_{0}$ are defined by the Fourier transform of the beam-beam potential (2.12) with account of the phase shift (2.13) as

$$
\begin{aligned}
& C_{\underline{m}}=2\left|H_{b \bar{b}}^{(m)}\right|, \quad \psi_{0}=\operatorname{Arg} H_{b \bar{b}}^{(\underline{m})}, \\
& H_{b \bar{b}}^{(m)}=\frac{1}{(2 \pi)^{4}} \iint e^{i n \theta-i \underline{m} \cdot \underline{\Psi}} H_{b b} d \theta d^{3} \underline{\Psi} .
\end{aligned}
$$

The synchrotron motion affects the resonance behavior in a number of ways. First, it somewhat weakens the principal resonance, $m_{s}=0$, by the virtue of: i) dephasing the successive beam-beam kicks in the result of chromatic tune modulation; ii) phase averaging of every kick due to a finite bunch length. On the other hand, these very same mechanisms give rise to the synchrotron satellites, $m_{s} \neq 0$, which can effectively widen the resonance if the Chirikov overlap condition is met. In addition to these mechanisms, the crossing angle and finite dispersion can contribute to excitation of the synchrotron satellites.

Let us consider these factors in some detail.

\subsection{Effect of finite crossing angle and dispersion}

Let us first consider the effect of finite crossing angle and dispersion ignoring for a while the chromaticity and finite bunch length. Using representation (2.14) we obtain in this case for the contribution of one IP

$$
\begin{aligned}
& H_{b \underline{b}}^{(m)}=\exp \left(i n \theta_{I P}+i \underline{m} \cdot \underline{\phi}_{I P}+i m_{s} \mu_{I P}\right) \frac{r_{p} N}{\pi^{4} \gamma} \frac{J_{x}^{\left|m_{x}\right| / 2}\left(r^{2} J_{y}\right)^{\mid m_{y} / / 2}\left(\kappa^{2} J_{s}\right)^{\mid m_{s} / / 2}}{\left(2\left|m_{x}\right|-1\right) ! !\left(2\left|m_{y}\right|-1\right) ! !\left(2\left|m_{s}\right|-1\right) ! !\left(1+\kappa^{2}\right)^{\left(\left|m_{x}\right|+\left|m_{s}\right|\right) / 2}} \times \\
& \int_{0}^{1} \frac{t^{\left|m_{x}\right|+\left|m_{y}\right|+\left|m_{s}\right|-1} d t}{\left[1+\left(r^{2}-1\right) t^{2}\right]^{\left(\left|m_{y}\right|+1\right) / 2}} X\left(0,\left|m_{x}\right|,\left|m_{s}\right|, \delta_{x} t, t \sqrt{\frac{J_{x}}{1+\kappa^{2}}}, t \sqrt{\frac{\kappa^{2} J_{s}}{1+\kappa^{2}}}\right) Y\left(0,\left|m_{y}\right|, \frac{\delta_{y} r t}{\sqrt{1+\left(r^{2}-1\right) t^{2}}}, \frac{r t \sqrt{J_{y}}}{\sqrt{1+\left(r^{2}-1\right) t^{2}}}\right)
\end{aligned}
$$

where the functions $X, Y$ are defined by eqs.(3.6), the phase $\mu_{s}$ is defined by the relations

$$
\sin \mu_{s}=\frac{D_{x} \sigma_{E}}{\sqrt{D_{x}^{2} \sigma_{E}^{2}+\alpha^{2} \sigma_{s}^{2}}}, \quad \cos \mu_{s}=\frac{\alpha \sigma_{s}}{\sqrt{D_{x}^{2} \sigma_{E}^{2}+\alpha^{2} \sigma_{s}^{2}}},
$$

also, we have dropped the constant phase common to all IPs.

Calculations for the Tevatron lattice show that effect of the horizontal dispersion at parasitic IPs is not significant even in the case of large synchrotron amplitudes characteristic to the superconducting $\mathrm{RF}$ parameters. Table 2 gives the strength $C_{\underline{m}}$ of the synchrotron satellites of the $m_{x}=5, m_{y}=0$ resonance at $J_{x}=16, J_{y}=0, J_{s}=1$ for the two RF systems. 


\begin{tabular}{|c|c|c|c|c|c|c|}
\hline$\left|m_{s}\right|$ & 0 & 1 & 2 & 3 & 4 & 5 \\
\hline $\mathrm{Cu}$ RF & $4.367 \cdot 10^{-12}$ & $4.103 \cdot 10^{-13}$ & $8.989 \cdot 10^{-14}$ & $1.717 \cdot 10^{-14}$ & $3.019 \cdot 10^{-15}$ & $4.266 \cdot 10^{-16}$ \\
\hline SC RF & $3.678 \cdot 10^{-12}$ & $8.453 \cdot 10^{-13}$ & $2.115 \cdot 10^{-13}$ & $2.859 \cdot 10^{-14}$ & $3.561 \cdot 10^{-14}$ & $1.919 \cdot 10^{-15}$ \\
\hline
\end{tabular}

Table 2. The strength $C_{\underline{m}}$ of the synchrotron satellites of the $5 Q_{x}$ resonance with two RF systems.

The effect of the dispersion on the strength of the principal resonance, $m_{s}=0$, also being rather small, we will exclude the horizontal dispersion from the following analysis (as well as the crossing angle which is not foreseen for the first stage of Run II).

\subsection{Finite bunch length effect on resonance excitation (phase averaging)}

Let us now consider the effect of rapid phase advance variation in the vicinity of low-beta IP on the resonance excitation. In the case of $\beta_{x}{ }^{*}=\beta_{y}{ }^{*}=\beta^{*}$ and of no offsets, no crossing angle nor dispersion at the IP, we can factorize the resonance coefficient introducing the longitudinal factor ${ }^{5}$

$$
L_{\underline{m}}\left(J_{s}\right)=\frac{1}{(2 \pi)^{2}} \iiint e^{-i m_{s} \psi_{s}-i\left(m_{x}+m_{y}\right) \varphi-i\left(m_{x} \eta_{x}+m_{y} \eta_{y}\right) \sigma} F_{L}\left(J_{s}^{\prime}\right) d J_{s}^{\prime} d \psi_{s} d \psi_{s}^{\prime},
$$

where $F_{L}$ is the normalized to unity distribution function in the longitudinal action variable, which we again choose to be Gaussian, $F_{L}=\exp \left(-J_{s}\right)$. It is obvious that $\left|L_{\underline{m}}\left(J_{s}\right)\right| \leq 1$ always.

In the case of a small bunch length, $\sigma_{s}<<\beta^{*}$, we may put $\varphi=-b$ and obtain (assuming $\eta_{x}=\eta_{y}=\eta=$ const for simplicity) the generalization of the Bessel satellite formula:

$$
L_{\underline{m}}\left(J_{s}\right)=\mathbf{J}_{m_{s}}\left[\left(m_{x}+m_{y}\right)\left(1-2 \beta^{*} \eta\right) \lambda \sqrt{J_{s}}\right] \cdot \exp \left[-\frac{1}{4}\left(m_{x}+m_{y}\right)^{2} \lambda^{2}\right]
$$

$\mathrm{J}_{n}(x)$ being the Bessel function and

$$
\lambda=\frac{\sigma_{s}}{\beta^{*} \sqrt{2}} .
$$

For infinitely short bunches, $\sigma_{s} \rightarrow 0$, the longitudinal factor is just the Kronecker delta: $L_{\underline{m}}\left(J_{s}\right)=\delta_{m s, 0}$.

It is interesting to note that while the finite bunch length provides most of the phase averaging described by the exponential factor in eq.(4.9), the chromaticity could suppress excitation of the synchrotron satellites at $\eta \beta^{*}=1 / 2$ or, recalling the definition $(2.8)$, at

$$
\frac{d}{d \delta_{p}} v_{x, y}=\frac{\alpha_{M} R}{2 \beta^{*}} \approx 4
$$

for the Tevatron parameters $\alpha_{M}=2.3 \cdot 10^{-3}, R=1 \mathrm{~km}, \beta^{*}=35 \mathrm{~cm}$. However, the large spread in the beambeam chromaticity makes such suppression impossible even for short bunches.

Let us continue analysis of an arbitrary bunch length case remembering that in the absence of the offsets only even-order resonances (and their satellites) can be excited by the beam-beam interaction:

$$
m_{x}+m_{y}=2 m
$$

Making notice that

\footnotetext{
${ }^{5}$ In fact it may depend on the transverse amplitudes due to the beam-beam contribution to the chromaticity related parameters $\eta_{x, y}$.
} 


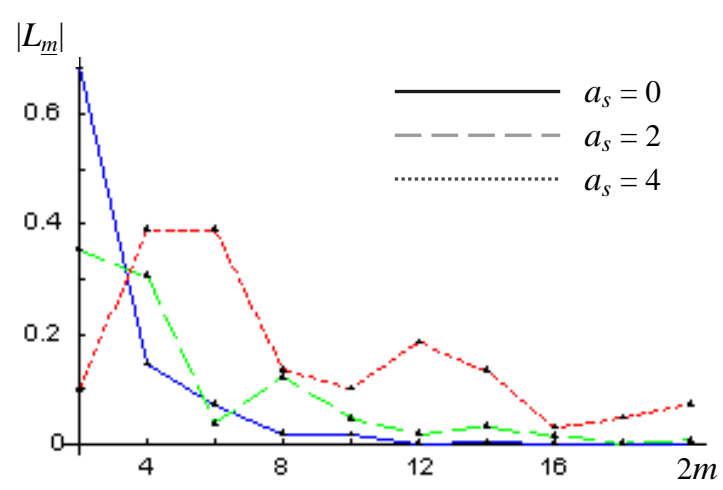

Figure 6. Longitudinal factor vs. resonance order

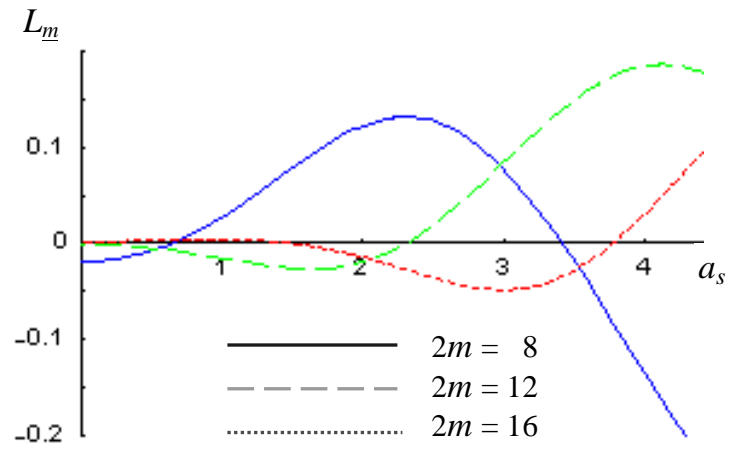

Figure 7. Longitudinal factor vs. synchrotron amplitude

$$
e^{-2 i m \varphi}=\left(\frac{2}{1+i \tan \varphi}-1\right)^{m}=(-1)^{m}\left[1+\int_{0}^{\infty} d t e^{-(1+i \tan \varphi) t} \sum_{k=1}^{m} \frac{(-2)^{k} m ! t^{k-1}}{(m-k) !(k-1) ! k !}\right],
$$

we can perform integration in (4.8) and obtain

$$
\begin{aligned}
L_{\underline{m}}\left(J_{s}\right)=(-1)^{m} & \left\{(-1)^{m_{s}} \mathbf{J}_{m_{s}}\left(4 m \beta^{*} \eta \lambda \sqrt{J_{s}}\right)+\right. \\
& \left.\int_{0}^{\infty} d t e^{-t-\lambda^{2} t^{2} / 4} \mathbf{J}_{m_{s}}\left[\lambda\left(t-4 m \beta^{*} \eta\right) \sqrt{J_{s}}\right] \sum_{k=1}^{m} \frac{(-2)^{k} m ! t^{k-1}}{(m-k) !(k-1) ! k !}\right\},
\end{aligned}
$$

For the graphical representation it is more convenient to introduce the normalized synchrotron amplitude, $a_{s}$, related to the normalized action variable by the formula (we assume the longitudinal emittances in the two beams to be equal)

$$
a_{s}=\sqrt{2 J_{s}}
$$

First we consider the finite bunch length effect on the principal resonance, $m_{s}=0$, at zero chromaticity, $\eta=0$. Fig. 6 shows the dependence of the absolute value of longitudinal factor (4.14) on the resonance order, $2 m$, for several values of the normalized synchrotron amplitude, $a_{s}$, at $\sigma_{s}=\beta^{*}$. For an on-momentum particle, $a_{s}=0$, the longitudinal factor rapidly falls off with the resonance order due to the phase averaging, reaching as small value as $10^{-3}$ at $2 m=12$. However, with increasing $a_{s}$ the phase averaging is less effective, as found in Ref.[6]. It may occur not sufficient to suppress the headon contribution to excitation of high order resonances.

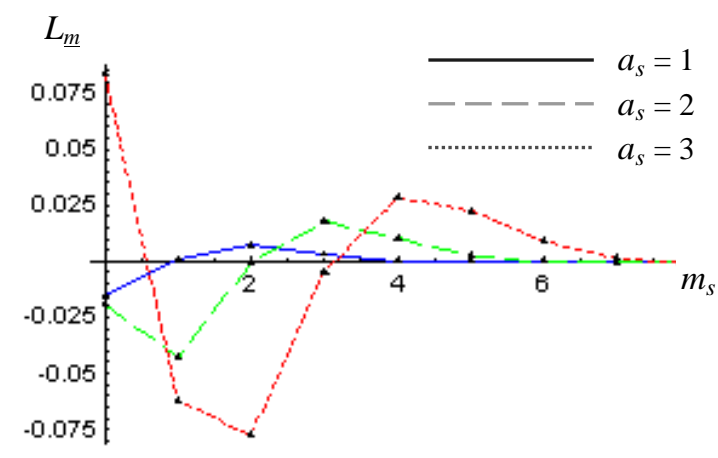

Figure 8. Longitudinal factor of $12^{\text {th }}$ order resonances vs. synchrotron satellite order.
Fig. 7 shows the longitudinal factor as a function of $a_{s}$ for the resonances of the order $2 m=8,12,16$, the dependence qualitatively reminds that found analytically in Ref.[6], though quantitatively it is rather different.

Now let us turn to the synchrotron sidebands, $m_{s} \neq 0$. Fig. 8 shows the dependence of the longitudinal factor of the $2 m=12$ resonances on the sideband number for several values of $a_{s}$ at $\sigma_{s} / \beta^{*}=1, \eta=0$. We see that the strength of the synchrotron satellites can be comparable with that of the principal resonance effectively increasing its width. Fig.9 shows beatings in the transverse amplitudes due to the $12^{\text {th }}$ order betatron 

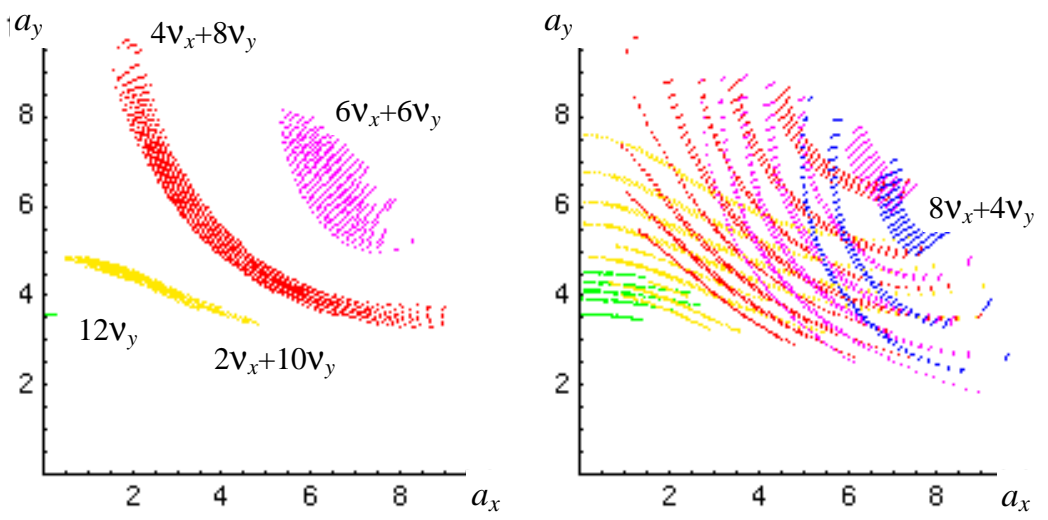

Figure 9. Swing of the transverse amplitudes due to the $12^{\text {th }}$ order resonances and their synchrotron satellites excited by the head-on interactions in the case of the existing RF (left) and SC RF (right).

resonances and their synchrotron satellites excited by the beam-beam interaction at two Tevatron lowbeta IPs at $J_{s}=1, \eta=0, \sigma_{s} / \beta^{*}=37 / 35, v_{s}=7 \cdot 10^{-4}$ (left) and $\sigma_{s} / \beta^{*}=14 / 35, v_{s}=6.4 \cdot 10^{-3}$ (right). In the first case the effect, although quite pronounced, should not pose significant problems in the Tevatron operation since the resonance groups are well separated. Let us note that the lines representing effect of a particular resonance are not shown if their length does not exceed a certain threshold value $(\sim$ the line width) or if they lie outside the circle $a_{\perp}=11$.

In the second case, which corresponds to the $20 \mathrm{MV}, 212 \mathrm{MHz} \mathrm{SC} \mathrm{RF}$, i) the width of the resonances becomes larger due decrease in the phase averaging, ii) satellites are farther apart due to a larger synchrotron tune. In the result the $12^{\text {th }}$ order resonances and their satellites form a maze through which particles, with some assistance from the external noise and higher order resonances, can escape starting from amplitudes possibly as low as $a_{\perp} \sim 4$.
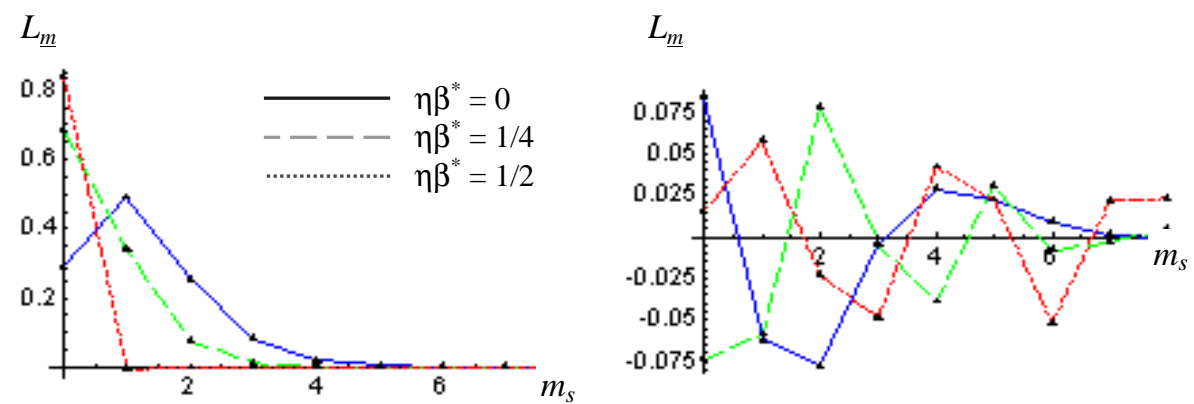

Figure 10. Longitudinal factor of $12^{\text {th }}$ order resonances vs. synchrotron satellite order at the indicated values of the chromaticity parameter $\eta$ and $\sigma_{s} / \beta^{*}=0.1$ (left) and $\sigma_{s} / \beta^{*}=1$ (right)

As noted earlier, in the case of a short bunch the chromaticity can (in principle) compensate the effect of the finite length on the synchrotron satellite excitation. It is no longer so in the case of a long bunch as illustrated by Fig.10 which shows the dependence of the longitudinal factor of the $12^{\text {th }}$ order resonances on the sideband number for $a_{s}=3$ and several values of $\eta$ at $\sigma_{s} / \beta^{*}=0.1$ (left) and $\sigma_{s} / \beta^{*}=1$ (right). In any case, this compensation will not work in Tevatron due to a large beam-beam chromaticity. 


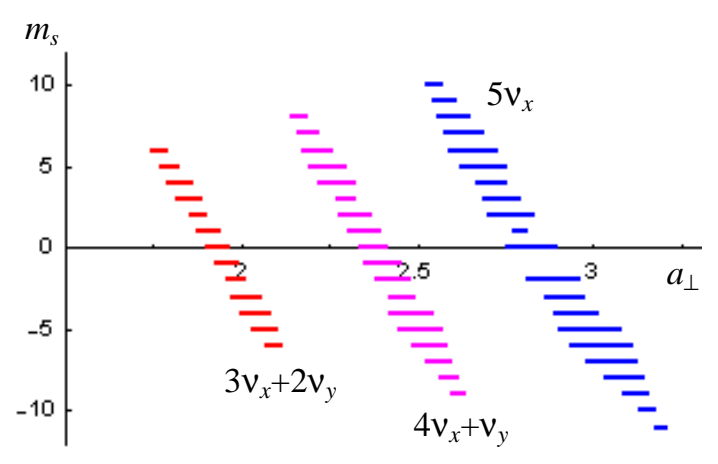

Figure 11. Swing of the transverse amplitude due to the $5^{\text {th }}$ order resonances and their synchrotron satellites at $\boldsymbol{a}_{s}=\sqrt{ } \mathbf{2}$.

\subsection{Betatron tune modulation}

At parasitic interaction points the betatron functions are usually so large in comparison with the bunch length that one may put $\varphi \rightarrow 0$ in eq.(4.8) and obtain the classical formula for the synchrotron satellites excitation by the betatron tune modulation

$$
L_{\underline{m}}\left(J_{s}\right)=\mathrm{J}_{m_{s}}\left[\frac{\left(m_{x} v_{x}^{\prime}+m_{y} v_{y}^{\prime}\right) \sigma_{E}}{v_{s}} \sqrt{2 J_{s}}\right]
$$

where the prime means differentiation of the tunes by $\delta_{p}$, the relation $\sigma_{s}=\beta_{s} \sigma_{E}$ was used.

Due to the large beam-beam contribution discussed in Section 3 the total chromaticity can not be made small by the conventional means of the sextupoles.

In the result the synchrotron satellites up to a high order,

$$
m_{s} \approx\left|\frac{m_{x} v_{x}^{\prime}+m_{y} v_{y}^{\prime}}{v_{s}}\right| \sigma_{E} a_{s},
$$

can be excited leading to an appreciable increase in the effective resonance width.

Fig.11 shows the swing of the transverse amplitude, $a_{\perp}$, defined by eqs.(3.8), due to the $5^{\text {th }}$ order resonances and their satellites excited by the long-range interactions for particles in the $6^{\text {th }}$ antiproton bunch with initially $a_{x}=a_{y}$ and $J_{s}=1\left(a_{s}=\sqrt{2}\right)$ in the absence of the bare lattice chromaticity $\left(\eta_{x 0}=\eta_{y 0}=0\right)$. The sidebands of different resonances overlap which means the possibility of fast particle transport over the entire set of resonances almost doubling the particle amplitude (from $a_{\perp} \approx 1.75$ to $a_{\perp} \approx 3.25$ ). This can affect not only the particle lifetime but the luminosity as well.
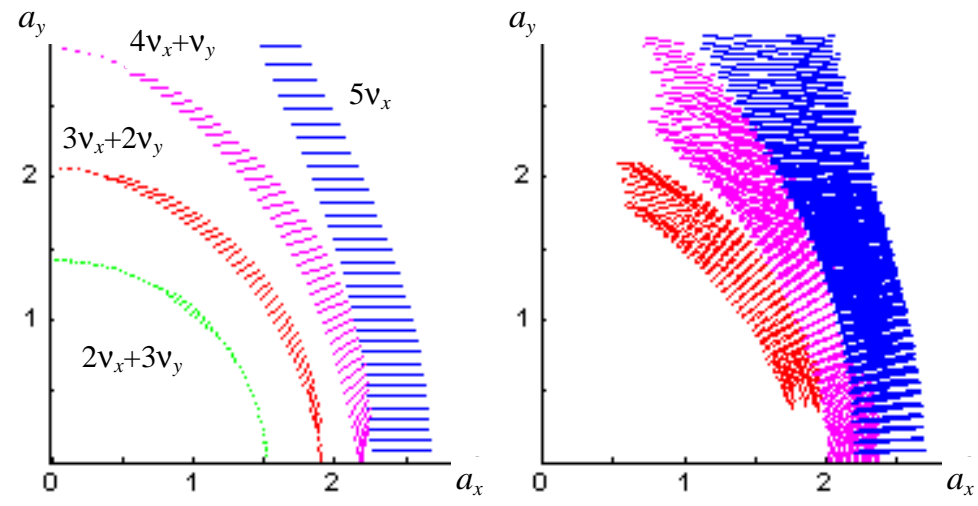

Figure 12. Swing of the transverse amplitudes due to the $5^{\text {th }}$ order resonances and their synchrotron satellites at $a_{s}=0$ (left) and $a_{s}=\sqrt{ } 2$ (right).

A 2D picture of the resonance satellites overlap is presented in Fig.12. The left plot shows the width of the betatron resonances for on-momentum particles $\left(J_{s}=0\right)$ when no satellite is excited. At large synchrotron amplitudes $\left(J_{s}=1\right)$ these resonances are, in accordance with eq.(4.16), noticeably suppressed (e.g. the $2 v_{x}+3 v_{y}$ resonance width falls below the chosen threshold). However, due to excitation of numerous satellites the effective resonance width is substantially increased.

With the superconducting RF the distance between the satellites of each of the $5^{\text {th }}$ order resonances becomes larger than the resonance width (Fig.13) but now the satellites of different 


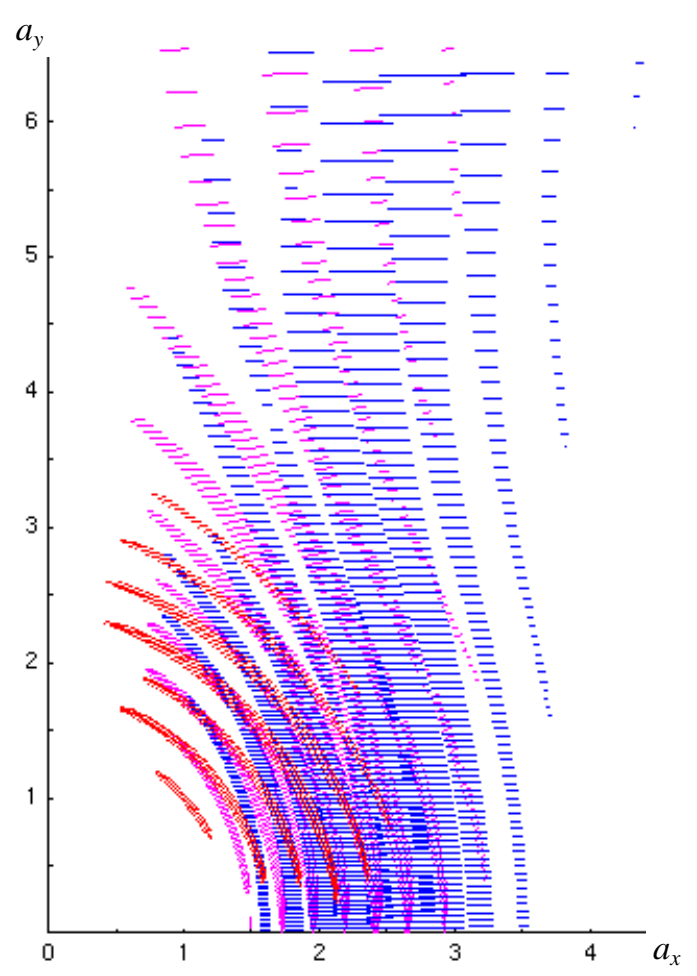

Figure 13. The same as in Fig.12 (right) but with the superconducting RF.

resonances overlap so that the amplitude range over which the fast diffusion can occur is further increasing.

\section{Summary}

The effects of the synchro-betatron coupling via the beambeam interaction appear to be quite significant in the Tevatron Run II configuration with $36 \times 36$ bunches, the main conclusions being:

- There is a strong beam-beam contribution to chromaticity rendering the total (intrabunch + bunch-tobunch) spread in chromaticity of $\sim 14$ units horizontally and $\sim 5$ units vertically.

- The chromatic tune modulation presents the major driving mechanism of the synchro-betatron resonances; the betatron phase modulation at low-beta IPs also provides a noticeable synchro-betatron coupling, whereas the effect of a finite dispersion at the parasitic IPs appears to be less important.

- With the standard bare lattice tunes, $v_{x 0}=20.585$, $v_{y 0}=20.575$, there is a strong excitation of the $5^{\text {th }}$ order resonances and their synchrotron satellites by the longrange beam-beam interactions leading to fast particle diffusion over the range of normalized betatron amplitudes from $a_{\perp} \approx 1.75$ to $a_{\perp} \approx 3.25$. This can affect not only the particle lifetime but the luminosity as well.

- Excitation of the satellites of the $12^{\text {th }}$ order resonances by the betatron phase modulation, although quite pronounced, should not pose significant problems in the Tevatron operation with the existing RF. However, with the planned upgrade to the superconducting RF it will lead to particle diffusion from amplitudes possibly as low as $a_{\perp} \sim 4$.

- It is necessary to extend the present analysis on the TEV33 configuration $(140 \times 121$ bunches, crossing angle, SC RF) where the effect of the odd-order resonances should be even stronger.

\section{Acknowledgements}

The author is grateful for the useful discussions of the subject of the present paper which he had over a stretch of time with W.Herr, J.Jowett, T.Sen and V.Shiltsev, who also read the manuscript and made a number of helpful remarks.

\section{References}

1. “Tevatron run II Handbook", http://www-bd.fnal.gov/runII/index.html

2. http://www-ap.fnal.gov/ martens/mad/tevatron/tevgen2.lat

3. B.Goddard, W.Herr, E.Keil et al., "Bunch Trains for LEP", CERN/SL/97-02 (AP), 1997; Particle Accelerators, v.57, p.237 (1998) 
4. S.Krishnagopal, R.Siemann, Phys. Rev. D, v.41, p.2312 (1990).

5. D.Pestrikov, "Synchrobetatron Resonances in Long Bunches due to Horizontal Crossing", in: Proc. VII Advanced ICFA Beam Dynamics Workshop, Dubna, 1995, pp. 77-90.

6. T.Sen, "The Beam-Beam Interaction of Finite Length Bunches in Hadron Colliders", FERMILABPub-00/093-T (2000).

7. K.Hirata, H.Moshammer and F.Ruggiero, "Synchro-Beam Interaction", CERN SL-AP/90-92 (1992), Particle Accelerators, v.40, p.205 (1993).

8. Y.Alexahin, "A Study of Coherent Beam-Beam Effect in the Framework of the Vlasov Perturbation Theory", LHC Project Report 461 (2001).

9. Y.Alexahin, "Analytical study of the incoherent beam-beam resonances in the Tevatron run II lattice with the beam-beam compensation", FERMILAB-PUB-00-120-T (2000).

10. J.Jowett, "Beam-beam kick and tuneshifts", http://wwwslap.cern.ch/ jowett/Mathematica/\#beambeam

11. Y.Alexahin, "On the Landau Damping and Decoherence of Transverse Dipole Oscillations in Colliding Beams", CERN SL-96-064 AP (1996); Particle Accelerators, v.59, p.43 (1998).

12. G.Lopez, "Head-on and Long Range Beam-Beam Tune Shift Spreads in the SSC", SSCL-Preprint304 (1993); Proc. PAC'93, Washington, 1993. 